\title{
Glucose-dependent downregulation of glucagon gene expression mediated by selective interactions between ALX3 and PAX6 in mouse alpha cells
}

\author{
Mercedes Mirasierra $^{1,2} \cdot$ Mario Vallejo ${ }^{1,2}$
}

Received: 15 June 2015 / Accepted: 7 December 2015 / Published online: 6 January 2016

(C) Springer-Verlag Berlin Heidelberg 2016

\begin{abstract}
Aims/hypothesis The stimulation of glucagon secretion in response to decreased glucose levels has been studied extensively. In contrast, little is known about the regulation of glucagon gene expression in response to fluctuations in glucose concentration. Paired box 6 (PAX6) is a key transcription factor that regulates the glucagon promoter by binding to the $\mathrm{G} 1$ and $\mathrm{G} 3$ elements. Here, we investigated the role of the transcription factor aristaless-like homeobox 3 (ALX3) as a glucose-dependent modulator of PAX6 activity in alpha cells.

Methods Experiments were performed in wild-type or Alx3-deficient islets and alphaTC1 cells. We used chromatin immunoprecipitations and electrophoretic mobility shift assays for DNA binding, immunoprecipitations and pull-down assays for protein interactions, transfected cells for promoter activity, and small interfering RNA and quantitative RT-PCR for gene expression.

Results Elevated glucose concentration resulted in stimulated expression of $A l x 3$ and decreased glucagon gene expression in wild-type islets. In ALX3-deficient islets, basal glucagon levels were non-responsive to changes in
\end{abstract}

Electronic supplementary material The online version of this article (doi:10.1007/s00125-015-3849-4) contains peer-reviewed but unedited supplementary material, which is available to authorised users.

Mario Vallejo

mvallejo@iib.uam.es

1 Centro de Investigación Biomédica en Red de Diabetes y Enfermedades Metabólicas Asociadas (CIBERDEM), Madrid, Spain

2 Instituto de Investigaciones Biomédicas Alberto Sols, Consejo Superior de Investigaciones Científicas/Universidad Autónoma de Madrid (CSIC/UAM), Calle Arturo Duperier 4, 28029 Madrid, Spain glucose concentration. In basal conditions ALX3 bound to the glucagon promoter at G3, but not at G1. ALX3 could form heterodimers with PAX6 that were permissive for binding to G3 but not to G1. Thus, increasing the levels of ALX3 in response to glucose resulted in the sequestration of PAX6 by ALX3 for binding to G1, thus reducing glucagon promoter activation and glucagon gene expression.

Conclusions/interpretation Glucose-stimulated expression of ALX3 in alpha cells provides a regulatory mechanism for the downregulation of glucagon gene expression by interfering with PAX6-mediated transactivation on the glucagon G1 promoter element.

Keywords Alpha cells · Glucagon · Glucose-dependent gene expression $\cdot$ Homeodomain $\cdot$ Paired domain $\cdot$ Transcription
Abbreviations
ALX3 Aristaless-like homeobox 3
ALX3 $\triangle \mathrm{C} \quad$ Truncated ALX3 excluding the carboxyl terminus
ALX3 $\Delta \mathrm{N}$ Truncated ALX3 excluding the amino terminus of ALX3
ALX3HD ALX3 homeodomain
ChIP Chromatin immunoprecipitation
EMSA Electrophoretic mobility shift assay
GST Glutathione $S$-transferase
PAX6 Paired box 6
PAX6PD Paired domain of PAX6
qPCR Quantitative real-time PCR
RNAi RNA interference
siRNA small interfering RNA 


\section{Introduction}

Beta cell dysfunction, accompanied by a reduced capacity of target tissues to respond to insulin, has largely been accepted as the main cause of chronic hyperglycaemia characteristic of diabetes $[1,2]$. In recent years, however, dysfunctional glucagon hypersecretion contributing to high blood glucose has been recognised as an additional and important aetiopathogenic factor [3, 4]. As hormonal stores depleted after secretion must be replenished by increasing glucagon gene expression and biosynthesis in a coordinated manner [5], the elucidation of the mechanisms that link glucagon gene transcription and glucagon secretion in response to fluctuations in glucose concentrations is of great importance for the understanding of the aetiopathogenic mechanisms of diabetes.

The secretion of glucagon from alpha cells stimulated in response to decreased blood glucose levels has been studied extensively [6-8]. In contrast, the study of glucose-regulated glucagon gene transcription has received less attention and the mechanisms involved remain unclear [9-12]. Glucagon gene transcription in alpha cells is regulated primarily by at least four promoter elements, termed G1-G4 [13-15], that are recognised by several transcription factors including paired box 6 (PAX6) [16-20]. PAX6 is particularly important because it is essential for the differentiation of alpha cells during development [21], and because it regulates the expression of the glucagon gene both directly [16, 22-24] and indirectly, acting on genes encoding transcription factors that, in turn, act on the glucagon promoter [25]. In addition, PAX6 regulates the expression of genes involved in prohormone processing [26] and glucagon secretion [27]. Therefore, PAX6 coordinately links glucagon production and secretion in alpha cells. However, it is unknown whether PAX6 or other G-element-binding transcription factors are involved in glucose-dependent regulation of glucagon gene expression.

In this study, we show that the homeodomain transcription factor aristaless-like homeobox 3 (ALX3), expressed in islets cells and previously found to be important for glucose homeostasis $[28,29]$, dynamically regulates glucose-dependent glucagon gene expression by engaging in protein-protein interactions with PAX6 that result in reduced PAX6 accessibility to the glucagon promoter in the presence of increased glucose concentrations.

\section{Methods}

Mice Alx3-deficient mice [30] derived from a colony originally provided by F. Meijlink (Netherlands Institute for Developmental Biology, Utrecht, the Netherlands), which has been maintained in our animal facilities for several years. Details about generation, maintenance and genotyping have been described previously [31]. Experiments were performed with 12-16-week-old male mice. Animals were randomly assigned to experiments as long as they satisfied inclusion criteria (age, sex and genotype). Experimental protocols were approved by the institutional bioethics committee and meet the requirements of current Spanish and European Union legislation.

Islets Mouse pancreatic islets were isolated as described previously [32]. Details are provided in the electronic supplementary material (ESM) Methods.

Glucagon content Batches of 30 islets were incubated overnight at $4{ }^{\circ} \mathrm{C}$ in $50 \mathrm{ml}$ lysis solution $(70 \%$ ethanol, $0.4 \% \mathrm{HCl}$ at $30 \%$, [all \% vol./vol.]). After centrifugation, the supernatant fraction was used for glucagon detection using an ELISA kit (YK090; Gentaur, Brussels, Belgium).

Quantitative RT-PCR Determinations were performed from total RNA samples as detailed in the ESM Methods and ESM Fig. 1. The sequences of the oligonucleotides used are shown in ESM Table 1.

Cell lines AlphaTC1 cells (alphaTC1-9, ATCC CRL-2350) (provided by I. Quesada, Miguel Hernandez University, Elche, Spain) were cultured as described previously [10], supplemented with $16 \mathrm{mmol} / \mathrm{l}$ glucose unless otherwise specified. BHK21 cells were cultured as described previously [28]. Cells were authenticated in our laboratory and checked to be free of mycoplasma contamination in our institutional cell culture facilities.

Electrophoretic mobility shift assays Electrophoretic mobility shift assays (EMSAs) were performed as described previously [33]. The sequences of the oligonucleotides used are shown in ESM Table 2. When indicated, ALX3 [33] or PAX6 (Millipore, MAB5552) antibodies were added to the binding reaction. In Fig. 3e, the ab64985 ALX3 antibody (Abcam, Cambridge, UK) was used.

Plasmids Alx3 and Pax6 expression plasmids have been described previously $[22,28]$. The oligonucleotides used for plasmid constructions are indicated in ESM Table 3. Luciferase reporter plasmids, plasmids encoding glutathione $S$-transferase (GST) fusion proteins and those used for in vitro transcription/translation are described in the ESM Methods.

Transfections Cells $\left(10^{5}\right.$ per well) were incubated with reporter plasmids $(0.5 \mu \mathrm{g})$ mixed with Transfectin (BioRad, Alcobendas, Madrid, Spain) in serum-free medium. Expression plasmids or the corresponding empty vectors were used when required, keeping the total amount of DNA constant. Luciferase activity was determined and corrected for transfection efficiency using the Renilla luciferase assay kit 
(Promega). The Rous sarcoma virus enhancer reporter plasmid RSV-Luc was used as a standard for normalisation. All transfections were performed in duplicates.

GST pull-down assays $\left[{ }^{35} \mathrm{~S}\right]$ Met-labelled and GST fusion proteins were used as described previously [33]. Details are provided in the ESM Methods.

Immunoprecipitation Immunoprecipitations [28] were performed using extracts prepared from alphaTC1 cells, and were followed by immunodetection of PAX6 or ALX3 by western blot, as indicated in the ESM Methods.

Chromatin immunoprecipitation assays Chromatin immunoprecipitation (ChIP) assays [28] were performed using chromatin extracted from mouse islets or alphaTC1 cells. Details are indicated in the ESM Methods and ESM Table 4.

RNA interference RNA interference (RNAi) [32] was performed using double-stranded RNA duplexes, as described in the ESM Methods.

Western blots Nuclear extracts from alphaTC1 cells and primary antibodies for ALX3 [34] (1:4,000 dilution), PAX6 (H-295, Santa Cruz Biotechnology, Heidelberg, Germany) (1:1,000 dilution) and actin (clone AC-15, Sigma, Madrid, Spain) (1:10,000 dilution) were used [32].

Statistical analysis The experimenter was blind to group assignment and outcome assessment in all experiments involving quantitative PCR determinations (RT-PCR, ChIP assays and RNAi). Results represent mean \pm SEM for the indicated number of experiments. Statistical significance was calculated using Student's $t$ test.

\section{Results}

Decreased glucagon levels in ALX3-deficient islets Reduced levels of glucagon content and gene expression in islets of ALX3-deficient mice (Fig. 1) [29] could not be attributed to decreased expression of key transcriptional regulators because Pax6 [16], Foxa2 [20] and Arx [35] did not change, whereas Mafb [17] increased (Fig. 1b). Thus, we hypothesised that ALX3 could regulate the glucagon promoter directly, and therefore fluctuations in the levels of ALX3 could contribute to the dynamic regulation of glucagon gene expression depending on the metabolic needs of the organism. To test this hypothesis we investigated whether the levels of ALX3 in alpha cells vary depending on fluctuations in glucose concentration.
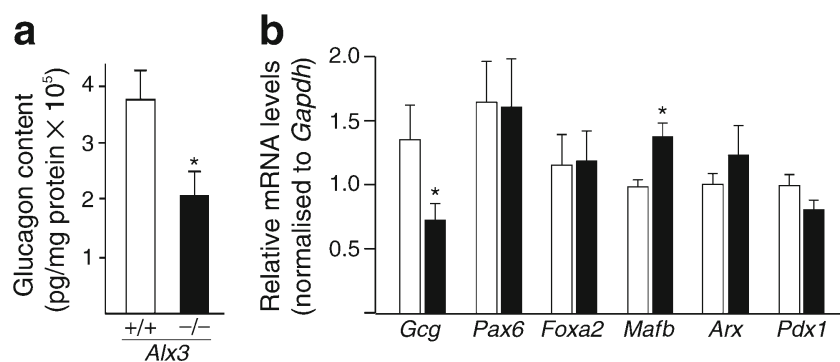

Fig. 1 Reduced glucagon levels in Alx3-deficient mice. (a) Glucagon content in isolated islets from wild-type or Alx3-deficient mice. $n=6$. (b) Relative mRNA levels of glucagon or alpha cell transcription factors and $P d x 1$ in islets of wild-type or Alx3-deficient mice. $n=5-15$. $* p<0.05$. +/+/white bars, wild type; -/-/black bars, Alx3 deficient

Expression of $A l x 3$ is stimulated by glucose and results in inhibition of glucagon gene expression The levels of Alx3 mRNA and ALX3 protein were elevated when alphaTC1 cells were cultured in the presence of high rather than low glucose, indicating that Alx3 is a glucose-responsive gene (Fig. 2a-c). We tested whether glucose-regulated Alx3 affects glucagon gene expression using cultured mouse islets. In wild-type islets, increasing the concentration of glucose in the medium resulted in a significant increase in Alx3 expression (Fig. 2d) and a concomitant decrease in glucagon expression (Fig. 2e). In contrast, in Alx3-deficient islets, increasing the concentration of glucose did not result in a significant change in the expression of glucagon mRNA (Fig. 2e). A glucosestimulated increase in insulin I expression was unaffected by lack of ALX3 (Fig. 2f).

ALX3 binds specific sites of the glucagon promoter EMSA with nuclear extracts from alphaTC1 cells and probes corresponding to the G3 or G1 sites showed that at least two of the bands detected on the $\mathrm{G} 3$ probe were disrupted by the addition of ALX3- or PAX6-specific antibodies (Fig. 3b). At the G1 site, the addition of the PAX6 antibody disrupted the formation of DNA-protein complexes, whereas the addition of the ALX3 antibody had no effect (Fig. 3c). The ALX3 antibody inhibited the formation of a complex on the $\mathrm{G} 1_{50}$ site (Fig. 3d), a homeodomain-binding site located downstream from G1 (Fig. 3a) [36]. Mutations in the PAX6 paired domain-binding site of G3 indicated that lack of binding of PAX6 prevented recruitment of ALX3 (ESM Fig. 2). ChIP assays demonstrated that both ALX3 and PAX6 are physically bound to the same region of the native glucagon promoter in vivo (Fig. 3e). These experiments indicate that ALX3 and PAX6 are part of the protein complex assembled on G3, and that the G1 site is bound by PAX6 as previously determined [36], but not by ALX3, which in turn recognises the downstream $\mathrm{G} 1_{50}$ site.

ALX3-PAX6 heterodimers bind G3 but not G1 To investigate whether ALX3 interacts directly with PAX6 we 
Fig. 2 Glucose-stimulated expression of $A l x 3$ reduces glucagon gene expression. (a, b) Levels of $A l x 3$ mRNA (a) and protein $(\mathbf{b})$ in alphaTC1 cells incubated in the presence of glucose at the indicated concentrations. In (b), the results of three independent experiments for each condition are shown. (c) Densitometric quantification of the ALX3 bands relative to actin bands shown in (b). (d-f) Levels of mRNA for $A l x 3$ (d), glucagon (e) and insulin I (f) in islets from wild-type or $A l x 3$-deficient mice incubated at the indicated concentrations of glucose $n=9-10 . * p<0.05$ and $* * p<0.01$. White bars, wild type; black bars, Alx 3 deficient a

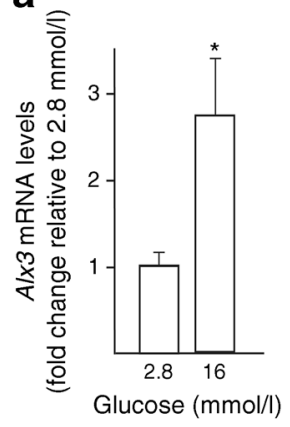

d

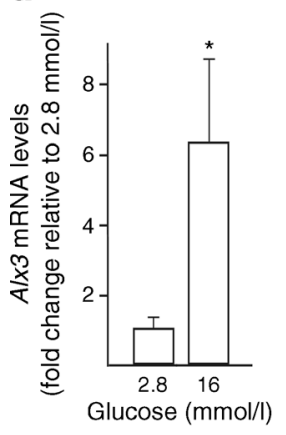

b

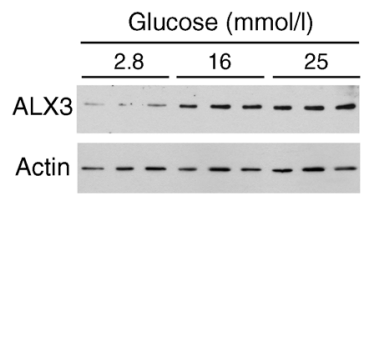

e

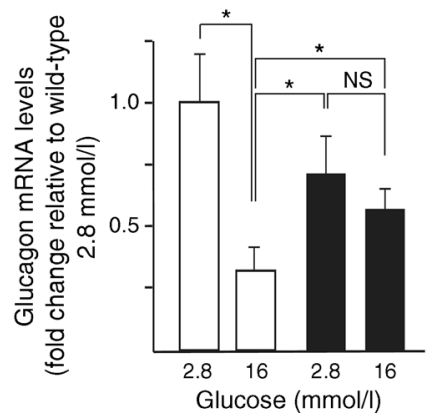

C

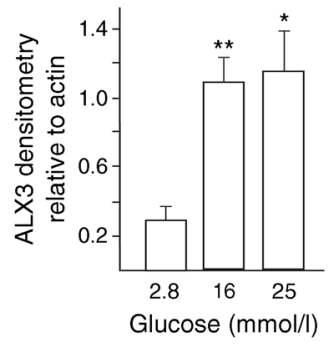

f

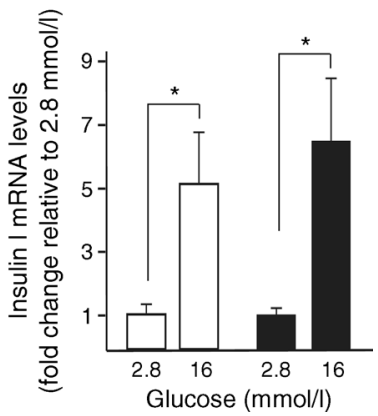

performed immunoprecipitations using alphaTC1 cells. PAX6 was detected in samples immunoprecipitated with an ALX3 antiserum (Fig. 4a), and vice versa (Fig. 4b), but not in those immunoprecipitated with control serum, indicating that ALX3 and PAX6 physically interact in the nuclei of alpha cells. The domains from each protein required for this interaction were studied using GST pull-down assays using full-length or truncated proteins (Fig. 4c). We found that $\left[{ }^{35} \mathrm{~S}\right]$ Met-labelled fulllength PAX6 was able to interact specifically with GST-ALX3 and with GST-ALX3 homeodomain (ALX3HD), but not with control GST or with GST fusion proteins containing either the carboxyl or the amino terminus of ALX3 (GST-ALX $3 \Delta \mathrm{N}$ and GST-ALX3 $\Delta$ C, respectively) (Fig. 4d). A similar pattern of interactions was found when the $\left[{ }^{35} \mathrm{~S}\right]$ Met-labelled paired domain of PAX6 (PAX6PD) was used (Fig. 4e). Finally, the $\left[{ }^{35} \mathrm{~S}\right]$ Met-labelled homeodomain of ALX3 (ALX3HD) could interact independently with either the paired domain or the homeodomain of PAX6 (GST-PAX6PD or GST-PAX6HD, respectively) (Fig. 4f). These results indicate that the homeodomain of ALX3 is sufficient for heterodimerisation via direct interactions with the paired domain or the homeodomain of PAX6.

The DNA binding consequences of these interactions were investigated using EMSA. PAX6 synthesised in vitro using a reticulocyte lysate could bind to the G3 probe as expected (Fig. 4g). In contrast, GST-ALX3HD, which bound efficiently to a previously described control probe (ESM Fig. 3a), failed to bind to G3 on its own. However, when the homeodomain of ALX3 was incubated together with PAX6 an additional band was generated, indicating that PAX6-ALX3 heterodimers can recognise G3 (Fig. 4g). In the case of the G1 site, binding of in vitro synthesised PAX6 (Fig. 4h) or PAX6 present in nuclear extracts of alphaTC1 cells (Fig. 4i) was confirmed by the addition of a specific antibody that disrupted binding. In both cases, the complexes containing PAX6 disappeared on the addition to the binding reaction of GST-ALX3, but remained undisturbed by the addition of control GST (Fig. 4h, i). Lack of binding of ALX3 to the G1 site was confirmed using recombinant ALX3 (ESM Fig. 3c, d).

These experiments indicate that ALX3-PAX6 heterodimers can bind to G3 efficiently, but are not capable of recognising G1. As G1 is a well-known target site for binding by PAX6, these results raise the possibility that increasing levels of ALX3 in alpha cells could result in inhibition of PAX6 binding to G1, thus resulting in decreased glucagon promoter activation. This notion was tested directly by transfections in alphaTC1 cells.

Functional interactions between ALX3 and PAX6 on the glucagon promoter Overexpression of ALX3 resulted in decreased luciferase activity elicited by the glucagon promoter reporter plasmid Gcg370Luc (Fig. 5a). In marked contrast, overexpression of PAX6 resulted in increased luciferase activity (Fig. 5a). When overexpressed together, the transactivation activity of PAX6 was inhibited by ALX3 in a concentrationdependent manner (Fig. 5b). In turn, increasing amounts of PAX6 were unable to transactivate the glucagon promoter in the presence of a fixed amount of ALX3 (Fig. 5b).

When using the Gcg370/205Luc reporter, including G3 but excluding G1, ALX3 stimulated luciferase activity to a similar 


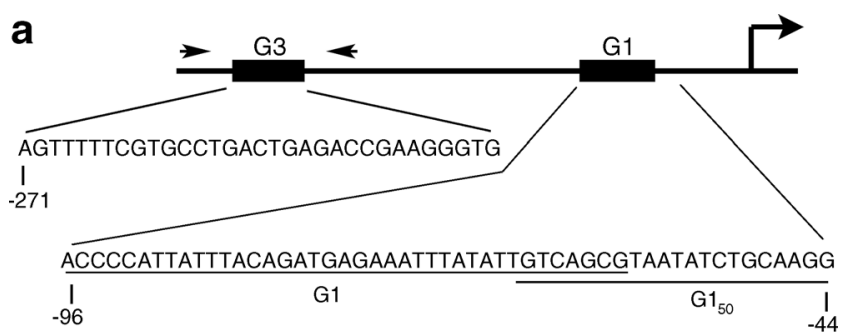

b

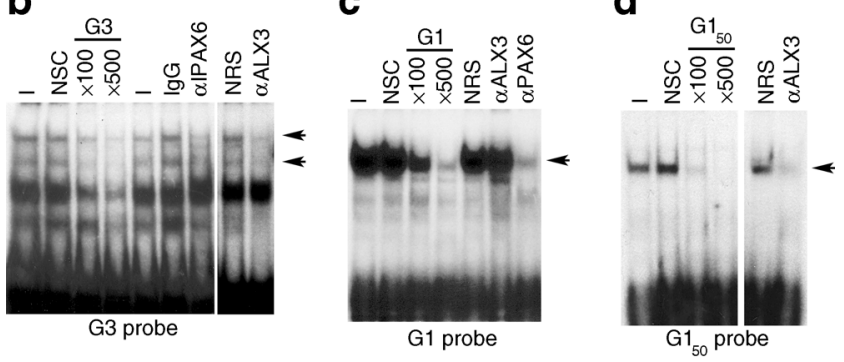

e

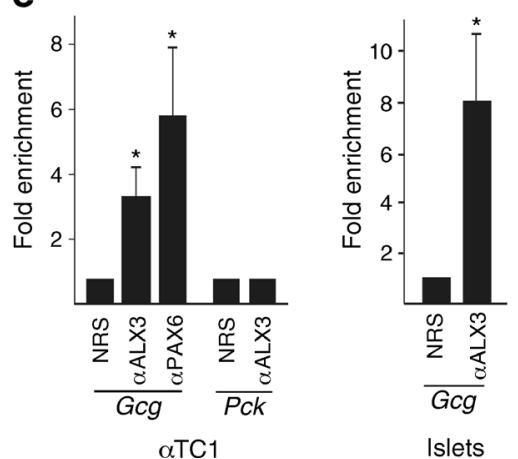

Fig. 3 ALX3 binds to the glucagon gene promoter. (a) Relative location of the G3, G1 and $\mathrm{G}_{50}$ sites in the glucagon promoter. Arrows indicate the location of primers used for quantitative real-time PCR (qPCR). (b-d) EMSAs showing the binding of nuclear proteins from alphaTC1 cells to G3 (b), G1 (c) and $\mathrm{G1}_{50}(\mathbf{d})$. The absence $(-)$ or presence of specific or non-specific competing (500-fold molar excess) oligonucleotides, or the addition of PAX6 or ALX3 antibodies, or control IgG or non-immune rabbit serum is indicated. Arrows indicate complexes containing PAX6 and/or ALX3. (e) ChIP assays analysed by qPCR showing amplification of a region of the glucagon promoter containing G3 immunoprecipitated with ALX3 or PAX6 antibodies, or with control non-immune rabbit serum from alphaTC1 cells or mouse islets. The phosphoenolpyruvate carboxykinase gene was used as negative control. ${ }^{*} p<0.05 ; n=3$. $\alpha$, antibody to; NSC, non-specific competing oligonucleotides; NRS, nonimmune rabbit serum

degree to that elicited by PAX6, and overexpression of both transcription factors concomitantly did not further increase or inhibit reporter activity (Fig. 5c). In contrast, when using Gcg122Luc, including G1 but excluding G3, overexpression of PAX6 stimulated luciferase activity, but overexpression of ALX3 decreased basal reporter activity and prevented transactivation by PAX6 (Fig. 5d). A similar pattern was found when using the reporter plasmids $3 \times \mathrm{G} 3 \mathrm{~T} 81 \mathrm{Luc}$, which carries three tandem copies of G3, or $3 \times$ G1T81Luc, which carries three tandem copies of G1, respectively (Fig. 5e, f). In BHK21 cells, which do not express ALX3 or PAX6 [33,
37], ALX3 did not increase Gcg370Luc activity on its own, but enhanced PAX6-dependent transactivation synergistically (ESM Fig. 4). However, this effect was reversed to an inhibition of luciferase activity with the highest amount of ALX3 used (ESM Fig. 4). These experiments support the notion that ALX3 contributes to glucagon promoter activity by acting on G3 co-ordinately with PAX6, and that, in turn, it is able to inhibit PAX6-dependent promoter activity on G1 when ALX3 levels increase.

Alx3 regulates glucose-dependent binding of PAX6 and glucagon expression in alpha cells Our data are consistent with a model according to which glucose-dependent decrease in glucagon gene expression could result from selective displacement of PAX6 from G1 as a consequence of the formation of new PAX6-ALX3 heterodimers generated in response to increased levels of ALX3 induced by glucose (Fig. 6). In alphaTC1 cells, glucose-dependent increase in ALX3 levels without affecting the levels of PAX6 (Fig. 6c) was accompanied by increased ALX3-PAX6 heterodimerisation demonstrated by immunoprecipitation (Fig. 6d). As predicted by our model, increased levels of ALX3 specifically induced by high glucose concentrations (Fig. 6e) were accompanied by decreased binding of PAX6 to G1 (Fig. 6f), whereas binding of PAX6 to G3 or of ALX3 to $\mathrm{G1}_{50}$, were unaffected (Fig. $6 \mathrm{~g}-\mathrm{h}$ ). Reduced binding to G1 was not observed when expression of Alx3 was silenced by small interfering (si)RNA (Fig. 6i-j).

We tested this model further in alphaTC1 cells with silenced expression of Alx3 (Fig. 7a-c). In the presence of $2.8 \mathrm{mmol} / 1$ glucose, glucagon expression was reduced in Alx3-knockdown cells relative to control cells (Fig. 7d), likely reflecting loss of ALX3 binding to $\mathrm{G} 3$ and $\mathrm{G} 1_{50}$ (Fig. 7e). In contrast, in the presence of $16 \mathrm{mmol} / \mathrm{l}$ glucose, glucagon expression increased in Alx3-knockdown cells relative to control cells (Fig. 7f), reflecting availability of unsequestered PAX6, a stronger transactivator, for binding to G1 (Fig. 7g). These experiments support the requirement of ALX3 for glucosedependent downregulation of glucagon gene expression in alpha cells.

\section{Discussion}

Our work indicates that ALX3 participates in the regulation of glucagon expression by binding with PAX6 to G3, and independently to $\mathrm{G1}_{50}$. PAX6 binds to $\mathrm{G} 1$ in the absence of ALX3, but PAX6-ALX3 heterodimers are unable to recognise G1. This has important functional implications because the levels of ALX3 are upregulated by glucose, thus favouring the formation of ALX3-PAX6 heterodimers engaging G1-bound PAX6. In this manner, ALX3 sequesters PAX6 and prevents its binding to $\mathrm{G} 1$, leading to downregulation of transcriptional activity. The inhibitory effect of ALX3 on G1-bound PAX6 is 

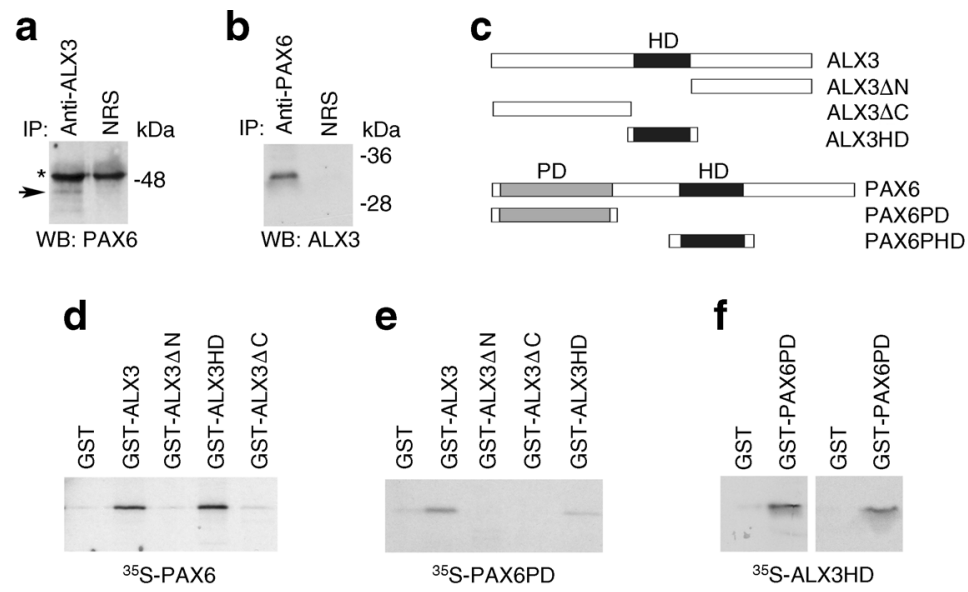

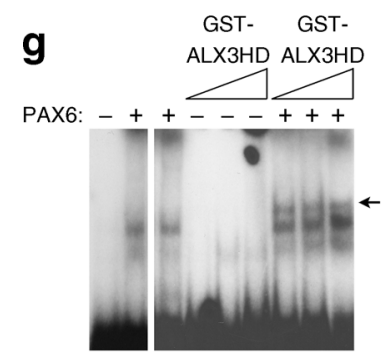

G3 probe

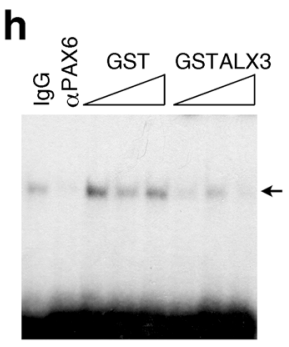

G1 probe IVT PAX6

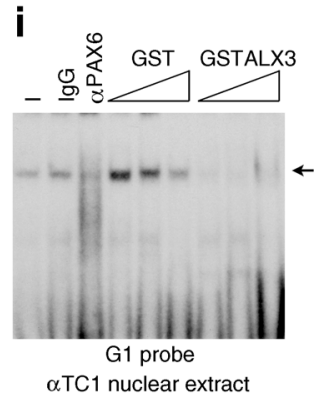

Fig. 4 Physical interactions and DNA binding of ALX3 and PAX6. (a, b) Western blots for PAX6 (a) or ALX3 (b) on samples immunoprecipitated from alphaTC1 cells with an ALX3 or a PAX6 antibody, respectively. The band showing PAX6 interacting with ALX3 is indicated by an arrow. (c) Full-length and truncated versions of ALX3 and PAX6 used in GST pull-down experiments. (d-f) GST pull-down experiments performed with ${ }^{35}$ S[Met]-labelled PAX6 (d), PAX6 paired domain (e), and ALX3 homeodomain (f). (g) EMSA with in vitro transcribed/translated PAX6 (+) or control reticulocyte lysate $(-)$ incubated

dominant because PAX6 is a stronger transactivator than ALX3. In agreement with this model, glucose-unregulated glucagon mRNA levels in ALX3-deficient islets are higher than those observed in control islets under high glucose conditions (see Fig. 2e and ESM Fig. 5). In ALX3-deficient islets, PAX6 would be bound to both G1 and G3 regardless of the concentration of glucose, whereas in wild-type islets, PAX6 would only be bound to G3, and the contribution of ALX3 bound to G3 and $\mathrm{G1}_{50}$ would be small because ALX3 is known to be a weak transactivator [28, 29] (see Fig. 5c, e).

Transcription factor interactions resulting in inhibition of glucagon expression by interfering with PAX6 have been described previously. Some of these interactions may have implications in the context of the pharmacological treatment of diabetes [24], or during embryonic development to prevent glucagon expression in non-alpha islet cells $[23,36,38]$, but their physiological relevance has not been completely established [39].

Glucagon secretion in the presence of reduced glucose concentration is coupled with stimulated transcription of the glucagon gene triggered by an auto-regulatory loop ensuring with radiolabelled G3 in the absence or presence of GSTALX3HD. Arrows indicate a PAX6-GSTALX3HD heterodimer. (h, i) Binding of in vitro transcribed/translated PAX6 (h) or alphaTC1 nuclear extracts (i) to radiolabelled G1. The complex containing PAX6 (arrow), identified by addition of a specific antibody, was inhibited by GSTALX3 but not by control GST. In (a): *non-specific bands. $\alpha \mathrm{TC} 1$, alpha TC1; HD, homeodomain; IVT, in vitro transcribed/translated; NRS, non-immune rabbit serum; $\mathrm{PD}$, paired domain

continuous hormone availability [5]. Our data support the existence of an ALX3-dependent counter-regulatory glucosesensing mechanism, acting at the transcriptional level, through which overproduction of glucagon would be prevented under conditions in which glucose concentration is elevated. Thus, downregulation of PAX6 function by glucose-induced modulation of the ALX3 to PAX6 ratio links transcriptional regulation of gene expression in alpha cells to fluctuations in the circulating concentrations of glucose to accommodate glucagon biosynthesis to the metabolic demands of the organism.

PAX6 links glucagon production and secretion by coordinating the expression of a gene network regulating these processes at different levels [16, 22-27]. Therefore, it is possible that these genes are regulated in a glucose-dependent manner via a mechanism involving selective interactions between PAX6 and ALX3 on different promoters. For example, the levels of $M a f b$ were elevated in islets from ALX3-deficient mice, suggesting that ALX3 inhibits Mafb expression. Although v-maf musculoaponeurotic fibrosarcoma oncogene family, protein B (MAFB) can act as a repressor [40], it is known to regulate glucagon gene expression [16, 17], and 
Fig. 5 Functional interactions between ALX3 and PAX6 on the glucagon promoter. Relative luciferase activities elicited in alphaTC1 cells cotransfected with Gcg370Luc (a, b), Gcg370/ 205Luc (c), Gcg122Luc (d), $3 \times$ G3T81Luc (e) or $3 \times$ G1T81Luc (f) reporter plasmids and the indicated amounts (ng) of expression plasmids encoding either ALX3 or PAX6. The relative positions of the $\mathrm{G} 3$ and/or G1 elements in the glucagon promoter are depicted on top of the histograms. $n=4-6$. ${ }^{*} p<0.05,{ }^{* *} p<0.01$ and $* * * p<0.001$ a
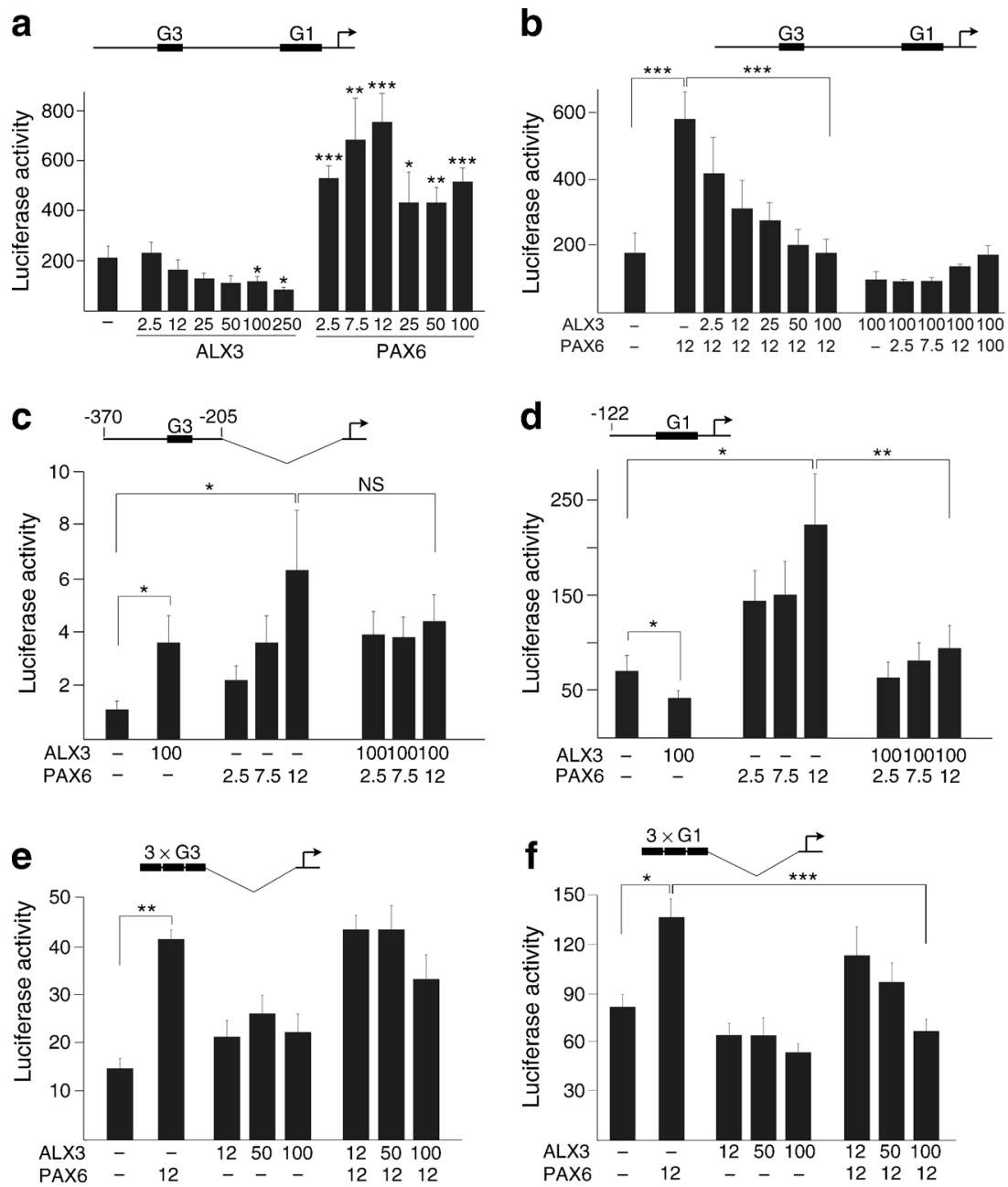

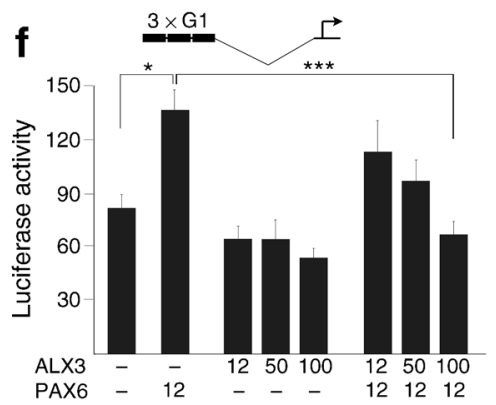

the Mafb promoter contains two potential PAX6-binding sites [25]. Thus, inhibition of Mafb expression by glucose-induced ALX3-mediated downregulation of PAX6 transactivation may provide an additional mechanism to reduce glucagon promoter activity in the presence of high glucose levels.

Apart from genes encoding transcription factors regulating glucagon gene expression [25], PAX6 regulates the expression of additional genes in alpha cells including those encoding the prohormone convertase 2 and its chaperone $7 \mathrm{~B} 2$, the free fatty acid receptor 1 (GPR40) and the glucosedependent insulinotropic peptide receptor (GIPR) [26, 27]. As these genes regulate glucagon production and secretion, the possible occurrence of glucose-dependent PAX6-ALX3 interactions on their promoters may be centrally important for the coordinated modulation of transcriptional mechanisms opposing glucagon overproduction under elevated glucose concentrations. This would place ALX3 as a key component of glucose-regulated glucagon production and secretion.

Both ALX3 and PAX6 contain paired-type homeodomains that can form dimers with selective DNA-binding specificities [33, 41, 42]. Besides, PAX6, but not ALX3, contains a paired domain. Both the paired domain and the homeodomain of
PAX proteins bind DNA, and in some cases they interact to promote cooperative binding to specific regulatory elements [43-45]. PAX6 heterodimerisation and alternative use of different protein subdomains modulate sequence selection and target site specificity of the binding partners [46]. In the glucagon promoter, the paired domain of PAX6 is sufficient for binding to G3, whereas the homeodomain is dispensable [47]. In contrast, both the paired domain and the homeodomain are essential for binding of PAX6 to G1 [47]. Therefore, PAX6 bound to G3 by the paired domain could tether ALX3 via interactions between their homeodomains, so that ALX3-PAX6 interactions involving homeodomain heterodimerisation would not disturb the paired domainmediated binding of PAX6 to G3, thus providing stable transactivation from this element. Indeed, the interaction between the ALX3 homeodomain and the PAX6 paired domain may even contribute to binding [43-45]. This type of interaction between PAX6 and other homeodomain transcription factors has been shown to be important for transcriptional regulation [48]. In contrast, as the paired domain is not sufficient for PAX6 binding to G1 [47], use of the G1-bound PAX6 homeodomain after recruitment of ALX3 for heterodimerisation 
a

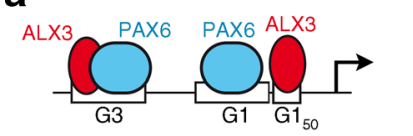

b

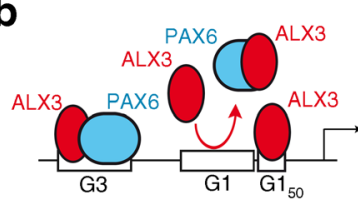

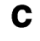

Glucose

$\mathrm{mmol} / \mathrm{l}$

$\stackrel{\infty}{\sim} \stackrel{\circ}{\circ}$

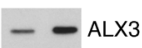

- - PAX6

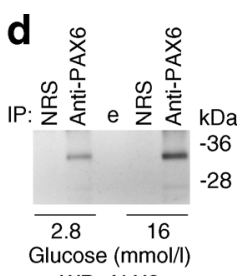

WB: ALX3

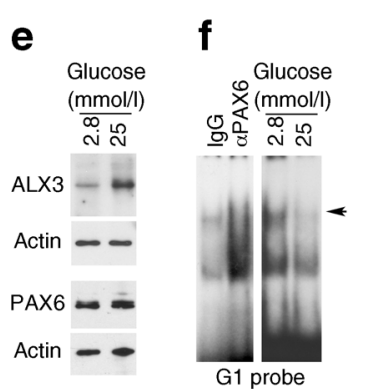

g

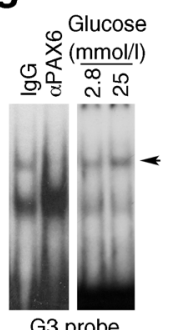

h

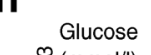

$\omega)(\mathrm{mmol} / \mathrm{l})$

뜬 i 늉

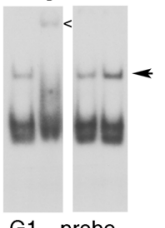

$\mathrm{G}_{50}$ probe
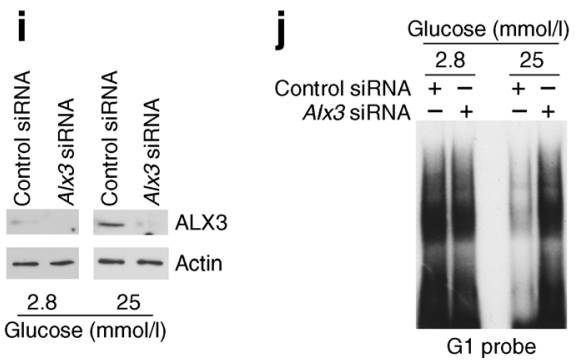

Fig. 6 ALX3 prevents binding of PAX6 to G1 in a glucose-dependent manner. (a, b) Hypothetical model of the glucose-dependent regulation of glucagon gene expression in alpha cells by ALX3 and PAX6: (a) basal; and (b) high glucose (high ALX3). (c) Western blot showing glucosedependent induction of ALX3 but not PAX6 in extracts of alphaTC1 cells used for immunoprecipitations (d). (d) Glucose-enhanced ALX3-PAX6 interaction shown by western blot for ALX3 on samples from alphaTC1 cells immunoprecipitated with a PAX6 antibody. (e) Western blot showing glucose-dependent induction of ALX3 but not PAX6 in extracts of alphaTC1 cells used in EMSA. (f-h) EMSA showing binding of nuclear extracts from alphaTC1 cells cultured at the indicated concentrations of glucose to $\mathrm{G} 1$ (f), G3 (g) or $\mathrm{G}_{50}(\mathbf{h})$. Arrows indicate the presence of PAX6 (f and $\mathbf{g}$ ) or ALX3 (h) identified by the addition of specific antibodies. In (h), the arrowhead indicates supershifted ALX3. (i) Western blot showing levels of ALX3 in alphaTC1 cells transfected with control or Alx3 siRNA. (j) EMSA showing binding to G1 of nuclear extracts from alphaTC1 cells transfected with control or Alx3 siRNA. In (i, j), glucose concentrations for cultured cells are indicated. In (d): e, empty lane; IP, immunoprecipitated; NRS, non-immune rabbit serum; WB, western blot

would preclude the use of PAX6 homeodomain for DNA binding, thus destabilising the protein-DNA interaction at this site.

The mechanism by which Alx3 expression is stimulated by glucose is unknown. In the case of intact islets, it cannot be excluded that insulin released from beta cells in response to glucose may contribute to downregulation of glucagon gene transcription. However, the results

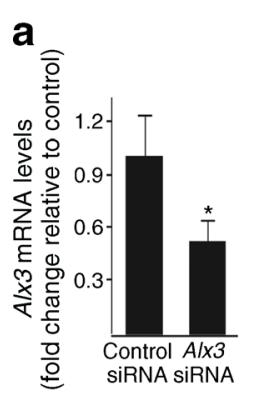

b

C

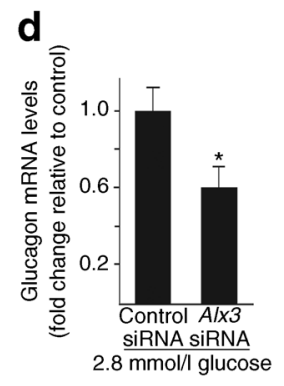

e
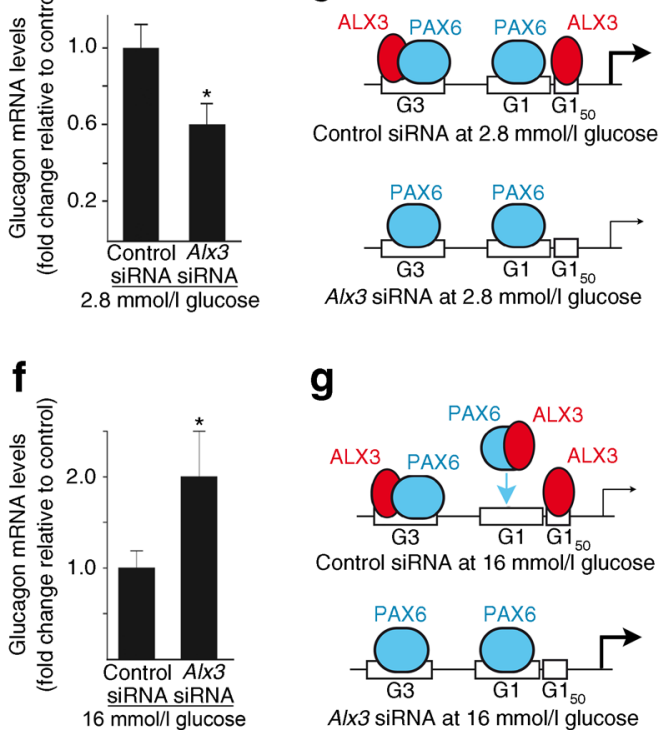

9
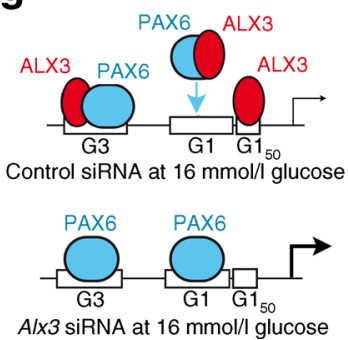

Fig. 7 Glucose-dependent regulation of glucagon expression by ALX3. (a) Levels of Alx3 mRNA in alphaTC1 cells transfected with control or Alx3 siRNA $(n=4)$. (b) Western blot showing levels of ALX3 in alphaTC1 cells transfected with control or Alx3 siRNA. Results from two representative experiments are shown. (c) Densitometric quantification of ALX3 bands relative to actin bands obtained from western blots similar to those shown in (b) $(n=6)$. $(\mathbf{d}-\mathbf{g})$ Relative levels of glucagon mRNA in alphaTC1 cells cultured at the indicated concentrations of glucose and transfected with control or Alx3 $\operatorname{siRNA}(n=4)(\mathbf{d}$ and f) and the respective schematic interpretation of the results in each condition (e and $\mathbf{g}) .{ }^{*} p<0.05$ and $* * p<0.01$

obtained from alphaTC1 cells, which retain important physiological features of intact alpha cells [5, 10, 49] strongly argue in favour of a direct effect of glucose on Alx3 expression.

Our findings may have implications for the aetiopathogenic mechanisms of diabetes. Dysfunctional glucagon hypersecretion contributing to hyperglycaemia has been recognised as an important factor in this disease [3, 4], suggesting the existence of impaired glucose-dependent inhibitory mechanisms affecting glucagon gene expression and hormone secretion. As ALX3 is important for the maintenance of glucose homeostasis in mice [29], it is possible that ALX3 loss-of-function mutations may contribute to dysregulated glucagon production in alpha cells. 
Acknowledgements We thank I. Quesada (Miguel Hernandez University, Elche, Spain) for providing alphaTC1 cells, F Meijlink (Netherlands Institute for Developmental Biology, Utrecht, the Netherlands) for providing the Alx3-deficient mice from which our colony was established, and A. Fernández-Perez for assistance with islet isolation for ChIP assays.

Funding This work was funded by the Spanish Ministry of Economy and Competitiveness (MINECO grants BFU2011-24245 and BFU201452149-R) and by the Instituto de Salud Carlos III. CIBERDEM is an initiative of the Instituto de Salud Carlos III.

Duality of interest The authors declare that there is no duality of interest associated with this manuscript.

Contribution statement MM performed the experiments. MV designed the study and wrote the paper. Both authors analysed the data and revised and approved the final version of the paper before submission, and are responsible for the integrity of this work. MV is the guarantor of this work.

\section{References}

1. Florez JC (2008) Newly identified loci highlight beta cell dysfunction as a key cause of type 2 diabetes: where are the insulin resistance genes? Diabetologia 51:1100-1110

2. Prentki M, Nolan CJ (2006) Islet $\beta$ cell failure in type 2 diabetes. J Clin Invest 116:1802-1812

3. Dunning BE, Gerich JE (2007) The role of $\alpha$-cell dysregulation in fasting and postprandial hyperglycemia in type 2 diabetes and therapeutic implications. Endocr Rev 28:253-283

4. Unger RH, Cherrington AD (2012) Glucagonocentric restructuring of diabetes: a pathophysiologic and therapeutic makeover. J Clin Invest 122:4-12

5. Leibiger B, Moede T, Muhandiramlage TP et al (2012) Glucagon regulates its own synthesis by autocrine signaling. Proc Natl Acad Sci U S A 109:20925-20930

6. Gromada J, Franklin I, Wollheim CB (2007) Alpha cells of the endocrine pancreas: 35 years of research and the enigma remains. Endocr Rev 28:84-116

7. Quesada I, Tudurí E, Ripoll C, Nadal A (2008) Physiology of the pancreatic $\alpha$-cell and glucagon secretion: role in glucose homeostasis and diabetes. J Endocrinol 199:5-19

8. Walker JN, Ramracheya R, Zhang Q, Johnson PRV, Braun M, Rorsman P (2011) Regulation of glucagon secretion by glucose: paracrine, intrinsic or both? Diabetes Obes Metab 13(Suppl 1): 95-105

9. Lee YS, Kobayashi O, Kikuchi O et al (2014) ATF3 expression is induced by low glucose in pancreatic $\alpha$ and $\beta$ cells and regulates glucagon but not insulin gene transcription. Endocr J 61:85-90

10. Marroquí L, Vieira E, González A, Nadal A, Quesada I (2011) Leptin downregulates expression of the gene encoding glucagon in alphaTC1-9 cells and mouse islets. Diabetologia 54:843-851

11. McGirr R, Ejbick CE, Carter DE et al (2005) Glucose dependence of the regulated secretory pathway in $\alpha \mathrm{TC} 1-6$ cells. Endocrinology 146:4514-4523

12. Dumonteil E, Magnan C, Ritz-Laser B, Ktorza A, Meda P, Philippe J (2000) Glucose regulates proinsulin and prosomatostatin but not proglucagon messenger ribonucleic acid levels in rat pancreatic islets. Endocrinology 141:174-180
13. Drucker D, Philippe J, Jepeal L, Habener JF (1987) Glucagon gene 5 '-flanking sequences promote islet cell-specific gene transcription. J Biol Chem 262:15659-15665

14. Morel C, Cordier-Bussat M, Philippe J (1995) The upstream promoter element of the glucagon gene, G1, confers pancreatic alpha cell-specific expression. J Biol Chem 270:3046-3055

15. Philippe J, Drucker D, Knepel W, Jepeal L, Misulovin Z, Habener JF (1988) Alpha-cell-specific expression of the glucagon gene is conferred to the glucagon promoter element by interactions of DNA-binding proteins. Mol Cell Biol 8:4877-4888

16. Gosmain Y, Avril I, Mamin A, Philippe J (2007) Pax-6 and c-Maf functionally interact with the $\alpha$-cell-specific DNA element G1 in vivo to promote glucagon gene expression. J Biol Chem 282: 35024-35034

17. Artner I, Le Lay J, Hang Y et al (2006) MafB: an activator of the glucagon gene expressed in developing islet $\alpha$ - and $\beta$-cells. Diabetes 55:297-304

18. Hussain MA, Miller CP, Habener JF (2002) Brn-4 transcription factor expression targeted to the early developing mouse pancreas induces ectopic glucagon gene expression in insulin-producing $\beta$ cells. J Biol Chem 277:16028-16032

19. Dumonteil E, Laser B, Constant I, Philippe J (1998) Differential regulation of the glucagon and insulin I gene promoters by the basic helix-loop-helix transcription factors E47 and BETA2. J Biol Chem 273:19945-19954

20. Lee CS, Sund NJ, Behr R, Herrera PL, Kaestner KH (2005) Foxa2 is required for the differentiation of pancreatic $\alpha$-cells. Dev Biol 278:484-495

21. St-Onge L, Sosa-Pineda B, Chowdhury K, Mansouri A, Gruss P (1997) Pax6 is required for differentiation of glucagon-producing $\alpha$-cells in mouse pancreas. Nature 387:406-409

22. Hussain MA, Habener JF (1999) Glucagon gene transcription activation mediated by synergistic interactions of pax- 6 and cdx- 2 with the p300 co-activator. J Biol Chem 274:28950-28957

23. Gauthier BR, Gosmain Y, Mamin A, Philippe J (2007) The $\beta$-cell specific transcription factor Nkx6.1 inhibits glucagon gene transcription by interfering with Pax6. Biochem J 403:593-601

24. Kratzner R, Frohlich F, Lepler K et al (2008) A peroxisome proliferator-activated receptor $\gamma$-retinoid X receptor heterodimer physically interacts with the transcriptional activator Pax6 to inhibit glucagon gene transcription. Mol Pharmacol 73:509-517

25. Gosmain Y, Marthinet E, Chaysac C et al (2010) Pax6 controls the expression of critical genes involved in pancreatic $\alpha$ cell differentiation and function. J Biol Chem 285:33381-33393

26. Katz LS, Gosmain Y, Marthinet E, Philippe J (2009) Pax6 regulates the proglucagon processing enzyme PC2 and its chaperone 7B2. Mol Cell Biol 29:2322-2334

27. Gosmain Y, Cheyssac C, Masson MH, Guerardel A, Poisson C, Philippe J (2012) Pax6 is a key component of regulated glucagon secretion. Endocrinology 153:4204-4215

28. Mirasierra M, Vallejo M (2006) The homeoprotein Alx3 expressed in pancreatic $\beta$-cells regulates insulin gene transcription by interacting with the basic helix-loop-helix protein E47. Mol Endocrinol 20:2876-2889

29. Mirasierra M, Fernández-Pérez A, Díaz-Prieto N, Vallejo M (2011) Alx3-deficient mice exhibit decreased insulin in beta cells, altered glucose homeostasis and increased apoptosis in pancreatic islets. Diabetologia 54:403-414

30. Beverdam A, Brouwer A, Reijnen M, Korving J, Meijlink F (2001) Severe nasal clefting and abnormal embryonic apoptosis in Alx3/Alx4 double mutant mice. Development 128:3975-3986

31. Lakhwani S, Garcia-Sanz P, Vallejo M (2010) Alx3-deficient mice exhibit folic acid-resistant craniofacial midline and neural tube closure defects. Dev Biol 344:869-880 
32. Fernández-Pérez A, Vallejo M (2014) Pdx1 and USF transcription factors co-ordinately regulate $\mathrm{Al} 3 \mathrm{3}$ gene expression in pancreatic $\beta$ cells. Biochem J 463:287-296

33. Pérez-Villamil B, Mirasierra M, Vallejo M (2004) The homeoprotein Alx3 contains discrete functional domains and exhibits cell-specific and selective monomeric binding and transactivation. J Biol Chem 279:38062-38071

34. García-Sanz P, Fernández-Pérez A, Vallejo M (2013) Differential configurations involving binding of USF transcription factors and Twist 1 regulate $A l x 3$ promoter activity in mesenchymal and pancreatic cells. Biochem J 450:199-208

35. Collombat P, Hecksher-Sorensen J, Krull J et al (2007) Embryonic endocrine pancreas and mature $\beta$ cells acquire $\alpha$ and PP cell phenotypes upon Arx misexpression. J Clin Invest 117:961-970

36. Ritz-Laser B, Gauthier BR, Estreicher A et al (2003) Ectopic expression of the beta-cell specific transcription factor Pdx1 inhibits glucagon gene transcription. Diabetologia 46:810-821

37. Gosmain Y, Katz LS, Masson MH, Cheyssac C, Poisson C, Philippe J (2012) Pax6 is crucial for $\beta$-cell function, insulin biosynthesis, and glucose-induced insulin secretion. Mol Endocrinol 26:696-709

38. Ritz-Laser B, Estreicher A, Gauthier BR, Mamin A, Edlund H, Philippe J (2002) The pancreatic beta-cell-specific transcription factor Pax-4 inhibits glucagon gene expression through Pax-6. Diabetologia 45:97-107

39. Flock G, Cao X, Drucker D (2005) Pdx-1 is not sufficient for repression of proglucagon gene transcription in islet or enteroendocrine cells. Endocrinology 146:441-449

40. Smink JJ, Tunn PU, Leutz A (2012) Rapamycin inhibits osteoclast formation in giant cell tumor of bone through the C/EBP $\beta$-MafB axis. J Mol Med 90:25-30
41. Tucker SC, Wisdom R (1999) Site-specific heterodimerization by paired class homeodomain proteins mediates selective transcriptional responses. J Biol Chem 274:32325-32332

42. Wilson DS, Sheng G, Lecuit T, Dostani N, Desplan C (1993) Cooperative dimerization of paired class homeodomains on DNA. Genes Dev 7:2120-2134

43. Jun S, Desplan C (1996) Cooperative interactions between paired domain and homeodomain. Development 122:2639-2650

44. Apuzzo S, Gros P (2007) Cooperative interactions between the two DNA binding domains of Pax3: helix 2 of the paired domain is in the proximity of the amino terminus of the homeodomain. Biochemistry 46:2984-2993

45. Xu HE, Rould MA, Xu W, Epstein JA, Maas RL, Pabo CO (1999) Crystal structure of the human Pax6 paired domain-DNA complexes reveals specific roles for the linker region and carboxyterminal subdomain in DNA binding. Genes Dev 13:1263-1275

46. Narasimhan K, Pillay S, Huang YH et al (2015) DNA-mediated cooperativity facilitates the co-selection of cryptic enhancer sequences by SOX2 and PAX6 transcription factors. Nucleic Acids Res 43:1513-1528

47. Grapp M, Teichler S, Kitz J et al (2009) The homeodomain of Pax6 is essential for PAX6-dependent activation of the rat glucagon gene promoter: evidence for a $\mathrm{PH} 0$-like binding that induces an active conformation. Biochim Biophys Acta-Gene Regul Mech 1789: 403-412

48. Mikkola I, Bruun JA, Holm T, Johansen T (2001) Superactivation of Pax6-mediated transactivation from paired domain-binding sites by DNA-independent recruitment of different homeodomain proteins. J Biol Chem 276:4109-4118

49. Tudurí E, Marroquí L, Soriano S et al (2009) Inhibitory effect of leptin on pancreatic $\alpha$-cell function. Diabetes 58:1616-1624 